\title{
The Effect of Different Enteral Feeding Protocols on Feeding Tolerance in Critically Ill Children
}

\author{
Nada Aljassim ${ }^{1}$, Sawsan Alyousef ${ }^{12^{*}}$, Muhammd Salman Bashir ${ }^{3}$, Mohamed Yousif ${ }^{1}$ and Noreen Mushtaq ${ }^{1}$ \\ ${ }^{1}$ Pediatric Critical Care Department, Specialized Children Hospital, King Fahad Medical City, Riyadh, Saudi Arabia \\ ${ }^{2}$ Center for Research, Education \& Simulation Enhanced Training (CRESENT), King Fahad Medical City, Riyadh, Saudi Arabia \\ ${ }^{3}$ Department of Biostatistics, Research Services Administration, Research Center, King Fahad Medical City, Riyadh,Saudi Arabia
}

Abstract
Objectives: To evaluate different enteral tube Feeding Protocols (FP) on feeding tolerance and achieving
the goal feeds (GF) duration in pediatric intensive care unit (PICU) among 2 groups of patients with
different risks of feeding intolerance.
Design: Prospective randomized study.
Settings \& Patients: The study is conducted in 20 bed capacity medical/surgical PICU at king Fahad
Medical City in Saudi Arabia over 1-year period. We included children from 1 month to 12 years old
who are admitted to PICU $>48$ hours after obtaining informed consent signed by either parent or legal
guardian. All patients with medical indication of admission were included except of gastroenterology
related illness.

Interventions: We categorize PICU patients into either low risk group or high-risk group because we think the severity of illness and medical management in the high-risk group, put them into a higher risk of early development of feeding intolerance. The patients were assigned into the high-risk group if they meet certain criteria that includes 1) need for inotropes/vasopressors $>24$ hours and/or 2) need for muscle relaxant infusion and/or 3) having severe metabolic acidosis with $\mathrm{PH}$ of less than 7.2, $\mathrm{HCO}_{3}$ serum level less than $10 \mathrm{mmol} / \mathrm{L}$ at the day of randomization to start the feeding. After assigning the patient into the risk group, then we blindly randomized each patient into one of the two feeding protocols, the continuous versus intermittent enteral NG/ OG tube feeding. Different variables were tracked to monitor the feeding tolerance of the feeding protocol like the frequency of vomiting and change in bowel motion. We determine the duration needed to reach the goal feeds recommended by a registered dietitian and we record any interruptions of the feeding protocol, duration of interruption and the reason.

Measurements and Main Results: Total of eighty-five patients were recruited in our study. Sixty-two patients in the low risk group ( 35 patients fed continuously \& 27 fed intermittently) and twenty-three patients in the high-risk group ( 11 patients fed continuously \& 12 intermittently). All patients were fed regular formula for age with goal feeds decided by registered dietitians. No significant differences in patients' characteristics. $65.7 \%$ of the low risk group who were continuously fed reached the goal feeds faster; within 2-3 days from feeding initiation vs $37 \%$ of the intermittent feeding group (P-value 0.025 ). $54.57 \%$ of the high-risk group who were continuously fed reached the goal feeds faster within 4-5 days vs $8.3 \%$ only of the intermittent feeding group (P-value 0.016 ). Both feeding protocols, continuous and intermittent feeding in both groups tolerate the feeds with no statistically differences. Feeding interruptions were not significantly different between both groups in each protocol with average interruption days of two days.

Conclusion: Implementation of the proposed continuous enteral tube feeding protocol significantly improve the delivery of nutrition in critically ill children in shorter duration than the intermittent boluses feeding protocol with good tolerance of both in different patients' risk groups. This is the first paper taking in consideration high risk factors because of the substantial risk of feeding intolerance that can affect early decision for feeding.

\section{Publication History:}

Received: November 07, 2017

Accepted: December 27, 2017

Published: December 29, 2017

\section{Keywords:}

Enteral feeding (EF), Feeding protocol (FP), Gastroesophageal reflux disease (GERD), Goal feeds (GF), Naso-Gastric (NG), Oro-Gastric (OG) Tube feeding, Pediatric intensive care unit (PICU).

\section{Introduction}

Malnutrition in children is a commonly encountered problem worldwide [1-3]. Malnutrition is also common among children admitted to the hospital especially in patients with chronic diseases. [3] So, Malnutrition can be preexisting problem or develop acutely with non-negligible effect of the critical illness $[4,5]$.

Critical illness is a risk of hyper catabolism and malnutrition $[6,7,8]$. The metabolic changes occur as initial response to stress lead to increased protein catabolism and loss of lean body mass, loss of functional proteins e.g. plasma proteins, enzyme systems and antibodies which interfere with the body immunity $[6,7,8]$. Use of medications in PICU like opioids [9], inotropes and vasopressors [10] can also be a potential factors associated with feeding intolerance and malnutrition during PICU stay. Vasopressors cause changes in the splanchnic blood flow to the gastrointestinal tract which may alter the patient tolerance to enteral feeds [10]. Fluid restriction, feeding ${ }^{*}$ Corresponding Author: Prof. Sawsan Alyousef, Center for Research, Education \& Simulation Enhanced Training (CRESENT), King Fahad Medical City; Riyadh Saudi Arabia; E-mail: salyousef@kfmc.med.sa

Citation: Alyousef S, Aljassim N, Bashir MS, Yousif M, Mushtaq N, et al. (2017) The Effect of Different Enteral Feeding Protocols on Feeding Tolerance in Critically III Children. Int J Pediatr Neonat Care 2: 134. doi: https://doi.org/10.15344/24552364/2017/134

Copyright: (c) 2017 Alyousef, et al. This is an open-access article distributed under the terms of the Creative Commons Attribution License, which permits unrestricted use, distribution, and reproduction in any medium, provided the original author and source are credited. 
Citation: Alyousef S, Aljassim N, Bashir MS, Yousif M, Mushtaq N, et al. (2017) The Effect of Different Enteral Feeding Protocols on Feeding Tolerance in Critically Ill Children. Int J Pediatr Neonat Care 2: 134. doi: https://doi.org/10.15344/2455-2364/2017/134

Page 2 of 9

interruptions prior performing procedures or interventions in critical care can also interfere with achieving nutrition. Malnutrition is associated with increasing morbidity $[11,12,13]$ and mortality in PICU [14]. Briassoulis et al. did a prospective study for 71 mechanically ventilated, critically ill children who received early enteral feeding through nasogastric tubes [11]. Only $22.7 \%$ of patients without protein deficiencies developed multiple organ system failure versus $37 \%$ of children who were at risk or already protein deficient [11]. Malnourished children stay longer on mechanical ventilatory support [12,13] which can increase the risk of infections and hospital stay [13]. In other studies mortality was also significantly associated with acute protein-energy malnutrition [14]. Hence, Early EF in PICU could reduce morbidities [15] and mortality [16,17]

Studies about achieving nutrition to critically ill patients have been the focus of researchers to improve the clinical practice and patients' outcome. American Society of Parenteral and Enteral Nutrition (A.S.P.E.N.) guidelines for the critically ill children recommended development of aggressive FP to enhance enteral nutrition delivery in shorter time [18]. Implementation of protocoled feeding approach is beneficial $[15,19,20,21]$. However, comparison between continuous and intermittent enteral feeding has been addressed in few studies without clear superiority $[22,23]$.

Our study objective is to evaluate implementation of different enteral tube feeding protocols for achieving the goal feeds as recommended by a registered dietitian among critically ill children and asses feeding tolerance. The feeding protocols are continuous and intermittent feeding in patients with different risk groups for feeding tolerance, low risk group and high-risk group. Feeding practice in our unit were either continuous or intermittent N/G or O/G feeding without following specific protocol, and switching from one method to another is internationally acceptable.

\section{Materials and Methods}

\section{Patients and setting}

We conducted a Prospective randomized study in PICU at king Fahad Medical City in Riyadh at Kingdom of Saudi Arabia with 20 medical/surgical bed capacity over 1-year period. We included All children who are equal or older than 1 month of age up to 12 years old with medical critical illness with estimated PICU stay of $>48$ hours. Informed consent was signed by either parent or legal guardian. We excluded patients who had surgical reason for the admission or postsurgery e.g. post craniotomy or fundoplication and patients who could feed by mouth, or had gastrostomy tube, ileostomy or colostomy. Also, we excluded patients who did not need gradual increment of feeding and could tolerate full feeds directly or been already on special (non regular) milk formula, patients with biliary and chronic liver diseases or with acute or chronic gastrointestinal diseases except isolated Gastro esophageal reflux disease (GERD).

\section{Feeding protocol}

We categorized PICU patients into low-risk group and highrisk group whenever the treating physician decide for starting tube feeding. High risk group patients should have one or more of the following criteria; first: being on vasopressors or inotropes for hemodynamic support for more than 24 hours at any doses (e.g. dopamine, epinephrine or norepinephrine infusion), second: being on muscle relaxant continuous infusion at any doses

Int J Pediatr Neonat Care

ISSN: 2455-2364 (e.g. rocuronium and cisatracurium), third: having severe metabolic acidosis at the day of randomization to start feeding; defined by low $\mathrm{PH}$ of less than 7.2 and serum Bicarbonate $\left(\mathrm{HCO}_{3}\right)$ level less than 10 $\mathrm{mmol} / \mathrm{L}$. The physician was blinded and randomly assign each patient into continuous or intermittent enteral feeding protocol through choosing from a labeled box for each risk group; high risk and low risk group. We used 5 blocks size randomization for each group. Then, the physician attached the feeding protocol and the stepwise management for any patient who develop intolerance in the patient chart for feeding initiation, advancement and specify the goal feeds as recommended by registered dietitians.

\section{Low risk group}

Patients who assigned to continuous feeding protocol received enteral tube feeding at rate of $0.5 \mathrm{ml} / \mathrm{kg} / \mathrm{hour}$ (hr) with maximum of $20 \mathrm{ml} / \mathrm{hr}$ as a starting infusion rate while those who were assigned to the intermittent boluses feeding protocol received feeding at volume of $1.5 \mathrm{ml} / \mathrm{kg}$ every 3 hours with maximum of $20 \mathrm{ml}$ bolus. The feeds were advanced with increment of $0.5 \mathrm{ml} / \mathrm{kg}$ Q6hrs in both groups till reach the goal feeds.

\section{High risk group}

Patients who were assigned to continuous feeding protocol received feeding at rate of $0.2 \mathrm{ml} / \mathrm{kg} / \mathrm{hr}$ with maximum of $10 \mathrm{ml} / \mathrm{hr}$ as a starting infusion rate while those who were assigned to the intermittent boluses feeding protocol received feeding at volume of $0.6 \mathrm{ml} / \mathrm{kg}$ every 3 hours with maximum of $10 \mathrm{ml}$ bolus. The feeds were advanced with increment of $0.2 \mathrm{ml} / \mathrm{kg}$ Q6hrs in both groups till reach the goal feeds.

The goal feeds for each patient was recommended by registered dietitians to achieve acceptable calories for patient's age with regards of the milk formula that each received. We excluded patients who required special (non-regular) milk formula or already on it from home. The feeding progress was checked daily by 2 different investigators collecting different variables including patient's demographics, diagnoses, medications receiving including inotropes, vasopressor, muscle relaxant, and anti-reflux medications and type of respiratory support used like mechanical ventilation or oxygen supplementation. These variables were recorded on a spreadsheet for the first 8 days after feeding initiation or up to 2 days after achieving goal feeds or until death or ICU discharge or transfer to the pediatric wards. We also tracked the number of days needed to reach the goal feeds and feeding interruptions with the rational. Any changes to the patient feeding protocol or event and management of feeding intolerance were documented. Indicators of feeding intolerance included the following; new onset vomiting (after exclusion of possibility of trigger like deep suctioning and inducing gag reflex), change in stools consistency or change in stools frequency, newly observed abdominal distension and gastric residual of $>20 \%$, prior feeds increment. Feeding intolerance was considered when the patient had 3 of the 5 indicators or isolated vomiting more than twice or isolated gastric residual. A stepwise management was applied for any patient developed intolerance. First step was to ensure patient having regular bowel movements by second day of feeding initiation and if not can use lactulose or glycerin suppositories and/or considering starting prokinetic agent; usually domperidone of $0.4-0.6 \mathrm{mg} / \mathrm{kg} /$ dose three times daily. If not tolerating feeds despite first step, the physician should proceed to the second step that was decreasing feeds rate by $50 \%$ of the running rate at the time of intolerance and 
Citation: Alyousef S, Aljassim N, Bashir MS, Yousif M, Mushtaq N, et al. (2017) The Effect of Different Enteral Feeding Protocols on Feeding Tolerance in Critically Ill Children. Int J Pediatr Neonat Care 2: 134. doi: https://doi.org/10.15344/2455-2364/2017/134

Page 3 of 9

continue advancing feeds as before. If still did not tolerate, third step was holding the feeds for 3 hours then resuming feeds at rate of $50 \%$ less than the previous feeding rate. Last, if did not tolerate feeds with no success of the three steps, physician should switch the feeding protocol from continuous feeding to intermittent boluses feed or vice versa.

\section{Outcome measures}

The objective for this study is to evaluate the achievement of goal feeds and assessing the feeding tolerance among PICU patients after implementation of NG/OG tube continuous feeding protocol vs intermittent bolus feeding protocol among low risk group patients and high-risk group.

\section{Sample size and statistical Analysis}

Sample size is calculated by the sample size software by considering 8 patients on average are admitted monthly to the PICU with the duration of more than 48 hours. Based on the average rate with $5 \%$ margin of error, $95 \%$ confidence limit and $80 \%$ power of test to detect such a difference at two-sided significance level. Therefore, the minimum estimated number of patients inflated by $10 \%$ loss of follow-up which accumulates to 85 patients needed in this study. 85 Patients were included in our study and consent was obtained from the parent/gradient. Each assigned into low/high risk then started on either continuous or intermittent feeding protocol randomly by block randomization (Figure 1). All Categorical variables gender, age group, reached full deed etc. were presented as numbers and percentages. Whereas continuous feeding interruption and number of days interruption were expressed as Mean \pm S.D. independent sample t-test was applied to determine the mean significant difference between two groups. Pearson's Chi-square/Fisher's exact test was applied according to whether the cell expected frequency is smaller than 5 and it was used to determine the significant association among categorical variables. $\mathrm{P}$ - value less than 0.05 was considered as statistically significant. All data was entered and analyzed through statistical package SPSS 22 (SPSS Inc., Chicago, IL, USA).

\section{Results}

\section{Low risk group}

Total of 62 patients were assigned in this group, 35 patients had continuous feeding protocol and 27 patients had intermittent feeding protocol. Underlying diseases of the patients in both groups were variable including chronic lung diseases with superimposed respiratory infection, bronchial asthma, aspiration pneumonia, seizures disorders, spinal muscular atrophy sepsis, sickle cell anemia, leukemia, solid tumors and immune deficiencies. General characteristics of the patients were similar between continuous feeding and intermittent feeding groups. There was statistical difference in number of days to reach the goal feeds between continuous group and intermittent group. $65.7 \%$ of continuously fed patients reached the goal feeds by $2-3$ days compared to $37.0 \%$ only of the intermittently fed patients $(\mathrm{p}=0.025)$.

No difference between patients in both protocols in the proportion of having GERD as diagnosis and use of anti-reflux medications; that is antacid drugs (ranitidine) or proton pump inhibitor (omeprazole) and prokinetic agent (domperidone). The average duration of feeds interruption was $2.08 \pm 1.82$ days in continuous feeding protocol vs $1.75 \pm 0.75$ days in intermittent feeding protocol $(\mathrm{P}=0.330)$ (Table1). 22 patients from both protocols suffered from feeds interruption that had delayed them from achieving the goal feeds in comparison to patients with no feeds interruptions. 13 patients from the continuous feeding protocol with mostly observed reason was feeding intolerance among 5 patients who responded to the management steps for feeding intolerance at step 3 or prior, 4 patients who were kept nil per oral (NPO) around the time of endotracheal tube extubation, 3 patients for performing body imaging or transfer to the images department and 1 patient had no clear reason. 9 patients in the intermittent feeding protocol had feeds interruptions for similar reasons, including 4 patients had feeding intolerance who responded to the management steps at the third step or prior except one patient that shifted to continuous feeding protocol, 4 patients were NPO around the extubation time and 1 patient for body imaging (Table 2).

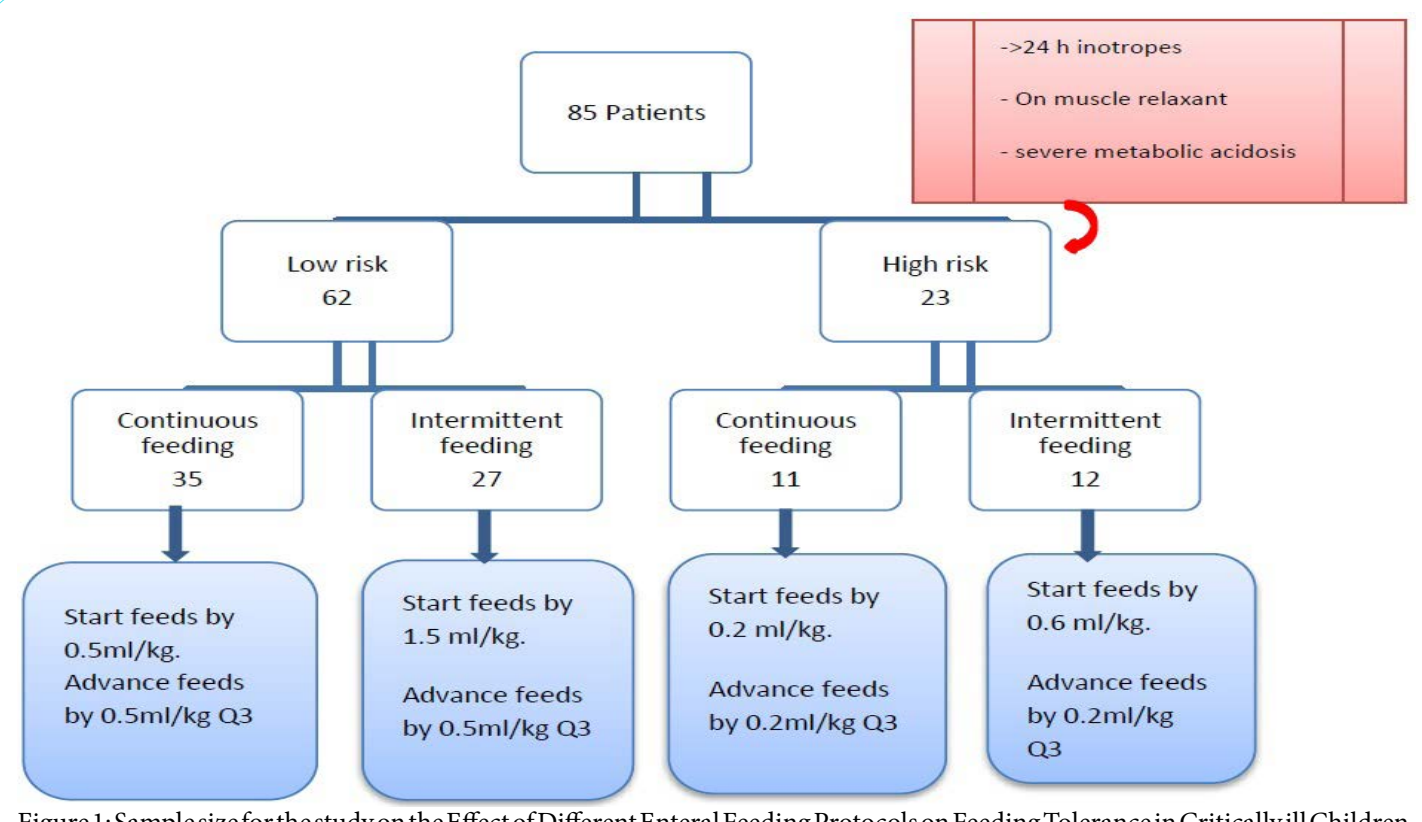

Figure 1: Sample size for the study on theEffect of Different Enteral Feeding Protocols on Feeding Tolerance in Criticallyill Children. 
Citation: Alyousef S, Aljassim N, Bashir MS, Yousif M, Mushtaq N, et al. (2017) The Effect of Different Enteral Feeding Protocols on Feeding Tolerance in Critically Ill Children. Int J Pediatr Neonat Care 2: 134. doi: https://doi.org/10.15344/2455-2364/2017/134

Page 4 of 9

In continuous feeding protocol we did not find differences between patients who had feeds interruption versus no interruption in stools frequency/consistency, but we observed patients in the intermittent feeding protocol who had no interruptions developed more frequent semi loose stool than patients who had feeds interruptions $(50.0 \%$ vs 33.3\%) but without statistical significance (Table 3 ).

\section{High risk group}

Total of 23 patients were assigned in this group and randomized into the feeding protocols. 11 patients were in the continuous feeding protocol and 12 patients were in the intermittent feeding protocol. Majority of underlying diseases were also respiratory infection, acute respiratory distress syndrome, asthma, seizures disorders, sepsis, shock with variable causes, tumors and immune deficiencies. There are 5 male patients (45.5\%) and 6 (54.5\%) female patients in the continuous feeding group while 3 males (25\%) and 9 females (75\%) patients in the intermittent feeding group. Patients in the continuous feeding protocol achieved the goal feeds faster than those in the intermittent feeding protocol again noted; $54.5 \%$ of the patients reached the goal feeds in $4-5$ days vs $8.3 \%$ in intermittent group ( $p$ value $=0.016$ )(Table 4). However, patients in the intermittent feeding protocol had more frequent semi loose stools $75.0 \%$ vs $45.5 \%$ in the continuous feeding. The average duration of feeds interruptions in intermittent feeding group was 2.33 days vs 1.75 days in the continuous group. 7 patients (63.6\%) the continuous feeding protocol had feeds interruption while 9 patients $(75 \%)$ from intermittent feeding protocol had interruption of feeds for different reasons (Table 5 and Table 6).

Interruption of feed affected the duration of achieving goal feeds. All patients with no feeds interruptions were on anti-reflux medications versus $42.9 \%$ of patients who had feeds interruption in the continuous feeding protocol (P-value of 0.058 ) with similar observation in the intermittent feeding protocol. (Table 7 and Table 8 )

\begin{tabular}{|c|c|c|c|c|}
\hline & & $\begin{array}{c}\text { Continuous } \\
\quad(\mathrm{n}=35)\end{array}$ & $\begin{array}{l}\text { Intermittent } \\
\quad(n=27)\end{array}$ & P-value \\
\hline \multirow{2}{*}{ Gender } & Male & $21(60.0 \%)$ & $16(59.3 \%)$ & \multirow{2}{*}{0.953} \\
\hline & Female & $14(40.0 \%)$ & $11(40.7 \%)$ & \\
\hline \multirow{3}{*}{ Age Group } & $<1$ year & $18(51.4 \%)$ & $13(48.1 \%)$ & 0.798 \\
\hline & $1-5$ years & $13(37.1 \%)$ & $10(37.0 \%)$ & 0.993 \\
\hline & $>5$ years & $4(11.4 \%)$ & $4(14.8 \%)$ & 0.693 \\
\hline \multirow{4}{*}{ Reach Full Feed } & $2-3$ days & $23(65.7 \%)$ & $10(37.0 \%)$ & ${ }^{*} 0.025$ \\
\hline & $4-5$ days & $9(25.7 \%)$ & $6(22.2 \%)$ & 0.750 \\
\hline & $>6$ days & $3(8.6 \%)$ & $0(0.0 \%)$ & 0.119 \\
\hline & Transfer to the ward & $0(0.0 \%)$ & $11(40.7 \%)$ & \\
\hline Feeding Interruption & & $13(37.1 \%)$ & $9(33.3 \%)$ & 0.795 \\
\hline No. of days Interruption & & $2.08 \pm 1.82$ & $1.75 \pm 0.75$ & 0.330 \\
\hline \multirow{7}{*}{ Stool Frequency/ Consistency } & 0 & $1(2.9 \%)$ & $0(0.0 \%)$ & 0.376 \\
\hline & 1-3 Formed & $7(20.0 \%)$ & $10(37.0 \%)$ & 0.136 \\
\hline & 1-3 Semi Loose & $17(48.6 \%)$ & $12(44.4 \%)$ & 0.745 \\
\hline & 3-5 Formed & $4(11.4 \%)$ & $2(7.4 \%)$ & 0.595 \\
\hline & 3-5 Semi Loose & $2(5.7 \%)$ & $2(7.4 \%)$ & 0.788 \\
\hline & $>6$ Formed & $2(5.7 \%)$ & $1(3.7 \%)$ & 0.715 \\
\hline & $>6$ Semi Loose & $1(2.9 \%)$ & $0(0.0 \%)$ & 0.376 \\
\hline \multirow[b]{2}{*}{ Medications } & Anti-reflux Medication & $25(71.4 \%)$ & $16(59.3 \%)$ & \multirow[b]{2}{*}{0.315} \\
\hline & Other Medication & $10(28.6 \%)$ & $11(40.7 \%)$ & \\
\hline \multirow{2}{*}{ Diagnosis } & GERD & $5(14.3 \%)$ & $2(7.4 \%)$ & \multirow[b]{2}{*}{0.396} \\
\hline & Without GRED & $30(85.7 \%)$ & $25(92.6 \%)$ & \\
\hline
\end{tabular}

Table 1: Continuous versus Intermittent Enteral Feeding Protocal for Low Risk Group

\begin{tabular}{|c|c|c|c|c|}
\hline & & $\begin{array}{c}\text { Continuous } \\
(\mathrm{n}=13)\end{array}$ & $\begin{array}{c}\text { Intermittent } \\
(\mathrm{n}=9)\end{array}$ & P-value \\
\hline \multirow{5}{*}{ Reasons for Interruption } & $\begin{array}{c}\text { Medical illness } \\
\text { (feeding intolerance) }\end{array}$ & $5(38.5 \%)$ & $4(44.4 \%)$ & 0.779 \\
\cline { 2 - 5 } & Pre/Post Extubation & $4(30.8 \%)$ & $4(44.4 \%)$ & 0.512 \\
\cline { 2 - 5 } & $\begin{array}{c}\text { Radiology } \\
\text { Investigation }\end{array}$ & $3(23 \%)$ & $1(11.1 \%)$ & 0.474 \\
\cline { 2 - 5 } & No clear reason & $1(7.7 \%)$ & $0(0.0 \%)$ & 0.394 \\
\hline
\end{tabular}

Table 2: Reason for feeding interruption among comtinuous and intermittent NG/OG enteral feeding for Low Risk group. 
Citation: Alyousef S, Aljassim N, Bashir MS, Yousif M, Mushtaq N, et al. (2017) The Effect of Different Enteral Feeding Protocols on Feeding Tolerance in Critically Ill Children. Int J Pediatr Neonat Care 2: 134. doi: https://doi.org/10.15344/2455-2364/2017/134

Page 5 of 9

\begin{tabular}{|c|c|c|c|c|}
\hline & & \multicolumn{2}{|c|}{ Continuous } & \multirow[t]{2}{*}{ P-value } \\
\hline & & $\begin{array}{l}\text { Interruption } \\
\quad(n=13)\end{array}$ & $\begin{array}{l}\text { Without Interruption } \\
\qquad(\mathrm{n}=22)\end{array}$ & \\
\hline \multirow[t]{2}{*}{ Gender } & Male & $9(69.2 \%)$ & $12(54.5 \%)$ & \multirow{2}{*}{0.392} \\
\hline & Female & $4(30.8 \%)$ & $10(45.5 \%)$ & \\
\hline \multirow[t]{3}{*}{ Age Group } & $<1$ year & $6(46.2 \%)$ & $12(54.5 \%)$ & 0.631 \\
\hline & $1-5$ years & $6(46.2 \%)$ & $7(31.8 \%)$ & 0.396 \\
\hline & $>5$ years & $1(7.7 \%)$ & $3(13.6 \%)$ & 0.593 \\
\hline \multirow[t]{3}{*}{ Reach Full Feed } & 2-3 days & $5(38.5 \%)$ & $18(81.8 \%)$ & ${ }^{*} 0.009$ \\
\hline & 4-5 days & $6(46.2 \%)$ & $3(13.6 \%)$ & ${ }^{*} 0.033$ \\
\hline & $>6$ days & $2(15.4 \%)$ & $1(4.5 \%)$ & 0.268 \\
\hline \multirow[t]{8}{*}{ Stool Frequency/ Consistency } & 0 & $0(0.0 \%)$ & $1(4.5 \%)$ & 0.435 \\
\hline & 1-3 Formed & $3(23.1 \%)$ & $4(18.2 \%)$ & 0.726 \\
\hline & 1-3 Semi Loose & $5(38.5 \%)$ & $12(54.5 \%)$ & 0.358 \\
\hline & 3-5 Formed & $1(7.7 \%)$ & $3(13.6 \%)$ & 0.593 \\
\hline & 3-5 Semi Loose & $2(15.4 \%)$ & $0(0.0 \%)$ & 0.058 \\
\hline & $>6$ Formed & $1(7.7 \%)$ & $1(4.5 \%)$ & 0.698 \\
\hline & $>6$ Semi Loose & $1(7.7 \%)$ & $0(0.0 \%)$ & 0.187 \\
\hline & $>6$ Loose & $0(0.0 \%)$ & $1(4.5 \%)$ & 0.435 \\
\hline \multirow[t]{2}{*}{ Medications } & $\begin{array}{l}\text { Anti-reflux } \\
\text { Medication }\end{array}$ & $9(69.2 \%)$ & $16(72.7 \%)$ & \multirow[t]{2}{*}{0.825} \\
\hline & Other Medication & $4(30.8 \%)$ & $6(27.3 \%)$ & \\
\hline \multirow[t]{2}{*}{ Diagnosis } & GERD & $1(7.7 \%)$ & $4(18.2 \%)$ & \multirow[b]{2}{*}{0.392} \\
\hline & Without GRED & $12(92.3 \%)$ & $18(81.8 \%)$ & \\
\hline
\end{tabular}

Table 3: Comparing Interruption versus no Interruption in Continuous Feeding Protocol for Low Risk Group

\begin{tabular}{|c|c|c|c|c|}
\hline & & \multicolumn{2}{|c|}{ Continuous } & \multirow[t]{2}{*}{ P-value } \\
\hline & & $\begin{array}{l}\text { Interruption } \\
\qquad(\mathrm{n}=9)\end{array}$ & $\begin{array}{l}\text { Without Interruption } \\
\qquad(\mathrm{n}=18)\end{array}$ & \\
\hline \multirow[t]{2}{*}{ Gender } & Male & $5(55.6 \%)$ & $11(61.1 \%)$ & \multirow[t]{2}{*}{0.778} \\
\hline & Female & $4(44.4 \%)$ & $7(38.9 \%)$ & \\
\hline \multirow[t]{3}{*}{ Age Group } & $<1$ year & $4(44.4 \%)$ & $9(50.0 \%)$ & 0.785 \\
\hline & $1-5$ years & $4(44.4 \%)$ & $6(33.3 \%)$ & 0.573 \\
\hline & $>5$ years & $1(11.1 \%)$ & $3(16.7 \%)$ & 0.702 \\
\hline \multirow[t]{3}{*}{ Reach Full Feed } & 2-3 days & $1(11.1 \%)$ & $9(50.0 \%)$ & 0.049 \\
\hline & 4-5 days & $5(55.6 \%)$ & $1(5.6 \%)$ & ${ }^{\star} 0.003$ \\
\hline & Transfer to the ward & $3(33.3 \%)$ & $8(44.4 \%)$ & 0.580 \\
\hline \multirow[t]{5}{*}{ Stool Frequency/ Consistency } & 1-3 Formed & $3(33.3 \%)$ & $7(38.9 \%)$ & 0.778 \\
\hline & 1-3 Semi Loose & $3(33.3 \%)$ & $9(50.0 \%)$ & 0.411 \\
\hline & 3-5 Formed & $1(11.1 \%)$ & $1(5.6 \%)$ & 0.603 \\
\hline & 3-5 Semi Loose & $1(11.1 \%)$ & $1(5.6 \%)$ & 0.603 \\
\hline & $>6$ Formed & $1(11.1 \%)$ & $0(0.0 \%)$ & 0.150 \\
\hline \multirow[t]{2}{*}{ Medications } & $\begin{array}{l}\text { Anti-reflux } \\
\text { Medication }\end{array}$ & $5(55.6 \%)$ & $11(61.1 \%)$ & \multirow[t]{2}{*}{0.782} \\
\hline & Other Medication & $4(44.4 \%)$ & $7(38.9 \%)$ & \\
\hline \multirow[t]{2}{*}{ Diagnosis } & GERD & $0(0.0 \%)$ & $2(11.1 \%)$ & \multirow[t]{2}{*}{0.299} \\
\hline & Without GRED & $9(100.0 \%)$ & $16(88.9 \%)$ & \\
\hline
\end{tabular}

Table 4: Comparing Interruption versus no Interruption in Intermittent Feeding Protocol for Low Risk Group 
Citation: Alyousef S, Aljassim N, Bashir MS, Yousif M, Mushtaq N, et al. (2017) The Effect of Different Enteral Feeding Protocols on Feeding Tolerance in Critically Ill Children. Int J Pediatr Neonat Care 2: 134. doi: https://doi.org/10.15344/2455-2364/2017/134

Page 6 of 9

\begin{tabular}{|c|c|c|c|c|}
\hline & & $\begin{array}{l}\text { Continuous } \\
(\mathrm{n}=11)\end{array}$ & $\begin{array}{l}\text { Intermittent } \\
\quad(\mathrm{n}=12)\end{array}$ & $\mathrm{P}$-value \\
\hline \multirow[t]{2}{*}{ Gender } & Male & $5(45.5 \%)$ & $3(25.0 \%)$ & \multirow{2}{*}{0.304} \\
\hline & Female & $6(54.5 \%)$ & $9(75.0 \%)$ & \\
\hline \multirow[t]{3}{*}{ Age Group(years) } & $<1$ & $5(45.5 \%)$ & $6(50.0 \%)$ & 0.827 \\
\hline & $1-5$ & $5(45.5 \%)$ & $6(50.0 \%)$ & 0.827 \\
\hline & $>5$ & $1(9.1 \%)$ & $0(0.0 \%)$ & 0.286 \\
\hline \multirow[t]{4}{*}{ Reach Full Feed } & 2-3 days & $1(9.1 \%)$ & $0(0.0 \%)$ & 0.286 \\
\hline & 4-5 days & $6(54.5 \%)$ & $1(8.3 \%)$ & ${ }^{\star} 0.016$ \\
\hline & $>6$ days & $4(36.4 \%)$ & $7(58.3 \%)$ & 0.292 \\
\hline & Transfer to the ward & $0(0.0 \%)$ & $4(33.3 \%)$ & 0.035 \\
\hline Feeding interruption & & $7(63.6 \%)$ & $9(75 \%)$ & 0.667 \\
\hline No. of days interruption & & $1.75 \pm 0.72$ & $2.33 \pm 0.87$ & 0.164 \\
\hline \multirow[t]{3}{*}{ Stool Frequency/ Consistency } & 1-3 Formed & $5(45.5 \%)$ & $3(25.0 \%)$ & 0.304 \\
\hline & 1-3 semi Loose & $5(45.5 \%)$ & $9(75.0 \%)$ & 0.147 \\
\hline & 3-5 semi Loose & $1(9.1 \%)$ & $0(0.0 \%)$ & 0.286 \\
\hline \multirow[t]{2}{*}{ Medications } & $\begin{array}{l}\text { Anti-reflux } \\
\text { Medication }\end{array}$ & $7(63.6 \%)$ & $6(50.0 \%)$ & \multirow[t]{2}{*}{0.51} \\
\hline & Other Medication & $4(36.4 \%)$ & $6(50.0 \%)$ & \\
\hline
\end{tabular}

Table 5: Continuous versus Intermittent Enteral Feeding Protocol for High Risk Group.

\begin{tabular}{|c|c|c|c|}
\hline \multicolumn{2}{|c|}{} & $\begin{array}{c}\text { Continuous } \\
(\mathrm{n}=7)\end{array}$ & $\begin{array}{c}\text { Intermittent } \\
(\mathrm{n}=9)\end{array}$ \\
\hline \multirow{3}{*}{ Reasons for interruption } & $\begin{array}{c}\text { Medical illness } \\
\text { (feeding intolerance) }\end{array}$ & $2(28.6 \%)$ & $2(22.2 \%)$ \\
\cline { 2 - 4 } & Pre/Post Extubation & $5(71.4 \%)$ & $6(66.6 \%)$ \\
\cline { 2 - 4 } & $\begin{array}{c}\text { Radiology } \\
\text { Investigation }\end{array}$ & $0(0.0 \%)$ & 0.771 \\
\hline
\end{tabular}

Table 6. Reasons for Interruption of feeds for Continuous versus Intermittent Enteral Feeding Protocol for High Risk Group.

\begin{tabular}{|c|c|c|c|c|}
\hline & & \multicolumn{2}{|c|}{ Continuous } & \multirow{4}{*}{$\begin{array}{c}\text { P-value } \\
0.303\end{array}$} \\
\hline & & $\begin{array}{l}\text { Interruption } \\
\quad(\mathrm{n}=7)\end{array}$ & $\begin{array}{l}\text { Without interruption } \\
\qquad(\mathrm{n}=4)\end{array}$ & \\
\hline \multirow[t]{2}{*}{ Gender } & Male & $4(57.1 \%)$ & $1(25.0 \%)$ & \\
\hline & Female & $3(42.9 \%)$ & $3(75.0 \%)$ & \\
\hline \multirow[t]{3}{*}{ Age Groups (years) } & $<1$ & $3(42.9 \%)$ & $2(50.0 \%)$ & 0.819 \\
\hline & $1-5$ & $3(42.9 \%)$ & $2(50.0 \%)$ & 0.819 \\
\hline & $>5$ & $1(14.3 \%)$ & $0(0.0 \%)$ & 0.428 \\
\hline \multirow[t]{3}{*}{ Reach goal Feed } & 2-3 days & $0(0.0 \%)$ & $1(25.0 \%)$ & 0.165 \\
\hline & 4-5 days & $3(42.9 \%)$ & $3(75.0 \%)$ & 0.303 \\
\hline & $>6$ days & $4(57.1 \%)$ & $1(25.0 \%)$ & 0.058 \\
\hline \multirow[t]{3}{*}{ Stool Frequency/ Consistency } & $1-3$ formed & $2(28.6 \%)$ & $3(75.0 \%)$ & 0.137 \\
\hline & 1-3 semi loose & $4(57.1 \%)$ & $1(25.0 \%)$ & 0.303 \\
\hline & 3-5 semi loose & $1(14.3 \%)$ & $0(0.0 \%)$ & 0.428 \\
\hline \multirow[t]{2}{*}{ Medication } & $\begin{array}{l}\text { Anti reflux } \\
\text { Medication }\end{array}$ & $3(42.9 \%)$ & $4(100.0 \%)$ & \multirow[t]{2}{*}{0.058} \\
\hline & Other Medication & $4(57.1 \%)$ & $0(0.0 \%)$ & \\
\hline
\end{tabular}

Table 7: Comparing Interruption versus no Interruption in Continuous Feeding Protocol for High Risk Group. 
Citation: Alyousef S, Aljassim N, Bashir MS, Yousif M, Mushtaq N, et al. (2017) The Effect of Different Enteral Feeding Protocols on Feeding Tolerance in Critically Ill Children. Int J Pediatr Neonat Care 2: 134. doi: https://doi.org/10.15344/2455-2364/2017/134

Page 7 of 9

\begin{tabular}{|c|c|c|c|c|}
\hline & & \multicolumn{2}{|c|}{ Intermittent } & \multirow[t]{2}{*}{ P-value } \\
\hline & & $\begin{array}{l}\text { Interruption } \\
\qquad(\mathrm{n}=9)\end{array}$ & $\begin{array}{l}\text { Without interruption } \\
\qquad(\mathrm{n}=3)\end{array}$ & \\
\hline \multirow[t]{2}{*}{ Gender } & Male & $2(22.2 \%)$ & $1(33.3 \%)$ & \multirow[t]{2}{*}{0.700} \\
\hline & Female & $7(77.8 \%)$ & $2(66.7 \%)$ & \\
\hline \multirow[t]{2}{*}{ Age Groups (years) } & $<1$ & $5(55.6 \%)$ & $1(33.3 \%)$ & 0.505 \\
\hline & $1-5$ & $3(42.9 \%)$ & $2(50.0 \%)$ & \\
\hline \multirow[t]{3}{*}{ Reach goal Feed } & 4-5 days & $0(0.0 \%)$ & $1(33.3 \%)$ & 0.070 \\
\hline & $>6$ days & $5(55.6 \%)$ & $2(66.7 \%)$ & 0.735 \\
\hline & Transfer to the ward & $4(44.4 \%)$ & $0(0.0 \%)$ & 0.157 \\
\hline \multirow[t]{2}{*}{ Stool Frequency/ Consistency } & $1-3$ formed & $3(33.3 \%)$ & $0(0.0 \%)$ & 0.248 \\
\hline & 1-3 semi loose & $6(66.7 \%)$ & $3(100.0 \%)$ & 0.248 \\
\hline \multirow[t]{2}{*}{ Medication } & Anti reflux Medication & $4(44.4 \%)$ & $2(66.7 \%)$ & 0.505 \\
\hline & Other Medication & $5(55.6 \%)$ & $1(33.3 \%)$ & \\
\hline
\end{tabular}

Table 8: Comparing Interruption and without interruption for Intermittent feeding protocol for High Risk Group.

\section{Discussion}

Nutrition is crucial for critically ill children. The awareness of nutrition as a therapy in critical care has been expanding $[18,24]$ with development of more feeding algorithms and protocols towards improvement of nutritional delivery $[15,19,20,21]$. Enteral feeding (EF) has advantageous effects of maintaining mucosal integrity of the gastrointestinal tract and preserving a complex immunological response $[25,26]$ that may contribute in protection from infections, in addition to the cost reduction. Early EF is associated with lower morbidity such as length of mechanical ventilation, length of hospital stays and increase the proportion of patients achieving goal feeds (GF) $[15,16,17]$.

Utilization of enteral feeding tubes is medically indicated because of difficulty to maintain normal oral diet in ill children for early EF initiation and providing appropriate energy requirements to prevent hypermetabolic state. The methods of EF via tubes are variable, two approaches are commonly used for gastric feeding; continuous feeding mostly around the clock and intermittent boluses for several times a day with no evidence of superiority [23]. Abdelsalam, et al. studied feeding protocol using both ways and showed using continuous feeding associated with higher delivery of nutrition on day 1 in comparison to the intermittent but this advantage disappeared by day 3 [23]. Also, wide diversity in feeding strategies exist in different pediatric and adult intensive care units and among practitioners within the same unit like specifying goal feeds, initiation timing, advancements volume and frequency. In addition to the fact of having frequent feeding interruptions related to procedures in PICU, performing of tests $[27,28,29]$ or presence of feeding intolerance at variable degrees. From clinical practice and previous observational studies, we hypothesized that high risk patients are those who are at higher risk of feeding intolerance and having higher chance of developing complications that lead to reluctance of practitioners to initiate EF. Mancl et al in a retrospective review of 259 adult patients received 346 episodes of concomitant $\mathrm{EF}$ and Intravenous vasopressor therapy for $>1$ hour, who had $74.9 \%$ tolerability to feeds with inverse relationship between maximum norepinephrine equivalent dose [30]. The Observed adverse events in the same study were rising serum lactate [30.6\%], elevated gastric residuals (14.5\%), emesis (9.0\%) and bowel ischemia/perforation (0.9\%) [30].
Critical illness is associated with elevation of cytokines release, glucocorticoids, insulin, glucagon, catecholamine in response to stress causing metabolic consequences and affect multiple body systems resulting in protein turnover, glucose intolerance, immune deregulation, capillary leak syndrome, edema and ileus $[6,7,31,32]$. These factors may contribute for developing feeding intolerance with respect to the difficulty of clinical judgment and diagnosis wither is real or perceived in addition to absence of sensitive marker for feeding intolerance at the current state [33].

In our study, we proposed 2 feeding protocols among 2 groups of patients differ in their risk of development of feeding intolerance. High risk group has patients who have one or more of the following factors at the time of randomization: treated with inotropes or vasopressor infusions at any doses for $>24$ hours, on muscle relaxant continuous infusion and/ or having metabolic acidosis with $\mathrm{PH}$ of less than 7.2, $\mathrm{HCO}_{3}$ serum level less than $10 \mathrm{mmol} / \mathrm{L}$. Also, we defined feeding intolerance by considering different variables that are used in clinical practice and we have a stepwise management if developed includes ensuring regular bowel movements and use of stool softeners or glycerin suppositories. The development of this intervention guide aims to minimize the diversity of practice and the frequency of feeds intolerance or interruptions that can compromise the goal achievement of consistent nutrition therapy to ill children. Also, Early prescription of a bowel regimen and limit constipation while avoiding diarrhea is recommended in American Society of Parenteral and Enteral Nutrition guidelines for 2009 [18].

Total of 85 patients were randomized in this study to follow one of the FP in both groups. 62 patients in the low risk group (35 patients in continuous FP \& 27 in intermittent FP) and 23 patients in the highrisk group (11 patients in continuous FP \& 12 in intermittent FP). The Patients have similar clinical characteristics with wide varieties of diagnoses. We found a significant difference among low risk group between continuous FP (65.7\%) and intermittent FP (37\%) in achieving the GF faster that is equivalent to 2-3 days (P 0.025) (Table1). Similar results found among high risk group with continuous feeding protocol was faster in achieving the goal feeds in shorter duration 4-5 days than intermittent feeding protocol, $54.5 \%$ versus $8.3 \%$ ( $\mathrm{P}$ value 0.016) (Table5). Most patients in low risk group tolerated the feeding protocol. 5 patients from continuous feeding protocol and 4 patients 
Citation: Alyousef S, Aljassim N, Bashir MS, Yousif M, Mushtaq N, et al. (2017) The Effect of Different Enteral Feeding Protocols on Feeding Tolerance in Critically Ill Children. Int J Pediatr Neonat Care 2: 134. doi: https://doi.org/10.15344/2455-2364/2017/134

Page 8 of 9

from intermittent feeding protocol developed feeding intolerance. Those patients had responded to the stepwise management before or at the 3rd step and one patient in the intermittent group had to switch to the continuous feeding protocol which was tolerated. Also, all patients in the high-risk group had tolerated the feeding except 2 from both $\mathrm{FP}$ and responded to the stepwise management of feeding intolerance. To our knowledge, these results are different from other studies that showed no difference between the continuous and intermittent FP in adult critical care clinically regarding duration of feed and tolerance [23] and inconclusive comparison in preterm infants from Cochrane review [34]. However, we found continuous FP faster to achieve the GF in children with equivalent tolerance compared to the intermittent FP. Interestingly, a recent review considering protein metabolism in acute illness in adults and children showed that continuous feeding persistently improves protein balance through a sustained suppression of protein breakdown from Kinetic studies in fed adults but studies were limited in pediatrics [22] and need further research with an accurate methodology to look into nitrogen balance and protein metabolism with different feeding methods.

Despite initiation of EF during our study, a variety of barriers impede maintenance of feeding during critical illness. Feeding interruptions were frequent related to medical illness and procedures but not significantly different in both groups between the two FP with average interruption of two days. Also, We observed that high proportion of patients are using anti-reflux medications without GERD diagnosis among patients which need further evaluation if it is related to the type of patient population that we have or our center clinical practice as we routinely prescribe Ranitidine to patients in PICU as ulcer prophylaxis as most PICU in the world. 4 patients in the high-risk group, continuous FP with no feeds interruptions $(100 \%)$ were on anti-reflux medications versus 3 patients who had feeds interruption (42.9\%) (P-value of 0.058) (Table 7) which may had contributed in faster reaching GF and perhaps better tolerance.

Our study has number of limitations. The results represent a single center experience of FP though our PICU is comparable to others. We did not track patients weight gain as most of patients' length of stay was less than 8 days and we only follow up the patient's tolerance of the protocol up to 2 days after achieving the goal feeds. The wide use of anti-reflux medications in our FP may bias the feeding tolerance results among the groups though it was not statistical significance between them.

\section{Conclusion}

Implementations of our proposed continuous enteral tube feeding protocol significantly improve the delivery of nutrition and achieving goal feeds in shorter duration than intermittent bolus feeds in critically ill children with good tolerance including high risk patients with efficacy of the feeding intolerance stepwise management. This is the first paper taking in consideration high risk factors in PICU that could potentially affect the nutrition delivery. However, the effect of these feeding protocols in protein metabolism and on long term outcomes has not been demonstrated and need to be further studied.

\section{Competing Interests}

The authors declare that they have no competing interests.

\section{References}

1. Rollet SR, Gray ES, Previl H, Forrester JE (2014) Prevalence of malnutrition in children under five and school-age children in Milot Valley, Haiti. Public Health 128: 1094-1098.

2. https://data.unicef.org/topic/nutrition/malnutrition

3. Joosten KF, Hulst JM (2008) Prevalence of malnutrition in pediatric hospital patients. Curr Opin Pediatr 20: 590-596.

4. Pollack MM, Wiley JS, Kanter R, Holbrook PR (1982) Malnutrition in critically ill infants and children. JPEN J Parenter Enteral Nutr 6: 20-24.

5. Merritt RJ, Suskind RM (1979) Nutritional survey of hospitalized pediatric patients. Am J Clin Nutr 32: 1320-1325.

6. Nunnally ME (2010) How Does Critical Illness Change Metabolism Evidence-Based Practice of Critical Care. Clifford S. Deutschman, Patrick J. Neligan 447-451

7. Newton LE, Heimburger DC (2006) Critical Illness. Handbook of Clinica Nutrition (Fourth Edition) 487-502

8. Mehta NM, Duggan CP (2009) Nutritional deficiencies during critical illness. Pediatr. Clin. North Am 56: 1143-1160.

9. Khansari M, Sohrabi M, Zamani F (2013) The Usage of Opioids and their Adverse Effects in Gastrointestinal Practice: A Review. Middle East J Dig Dis 5: 5-16.

10. Wells DL (2012) Provision of Enteral Nutrition During Vasopressor Therapy for Hemodynamic Instability: An Evidence-Based Review. Nutr Clin Pract 27: $521-526$

11. Briassoulis G, Zavras N, Hatzis T (2001) Malnutrition, nutritional indices, and early enteral feeding in critically ill children. Nutrition 17: 548-557

12. Grippa RB, Silva PS, Barbosa E, Bresolin NL, Mehta NM, et al. (2017) Nutritional status as a predictor of duration of mechanical ventilation in critically ill children. Nutrition 33: 91-95.

13. de Souza Menezes F, Leite HP, Koch Nogueira PC (2012) Malnutrition as an independent predictor of clinical outcome in critically ill children. Nutrition 28: 267-270

14. Pollack MM, Ruttimann UE, Wiley JS (1985) Nutritional Depletions in Critically III Children: Associations with Physiologic Instability and Increased Quantity of Care. JPEN J Parenter Enteral Nutr 9: 309-313

15. Briassoulis GC, Zavras NJ, Hatzis MD TD (2001) Effectiveness and safety of a protocol for promotion of early intragastric feeding in critically ill children. Pediatr Crit Care Med 2: 113-121.

16. Mikhailov TA, Kuhn EM, Manzi J, Christensen M, Collins M, et al. (2014) Early Enteral Nutrition Is Associated With Lower Mortality in Critically III Children. JPEN J Parenter Enteral Nutr 38: 459-466.

17. Khalid I, Doshi P, DiGiovine B (2010) Early Enteral Nutrition and Outcomes of Critically III Patients Treated With Vasopressors and Mechanical Ventilation. Am J Crit Care 19: 261-268.

18. Mehta NM, Compher C, A.S.P.E.N. Board of Directors (2009) Clinica Guidelines: nutrition support of the critically ill child. JPEN J Parenter Enteral Nutr 33:260-276.

19. Petrillo-Albarano T, Pettignano R, Asfaw M, Easley K (2006) Use of a feeding protocol to improve nutritional support through early, aggressive, enteral nutrition in the pediatric intensive care unit. Pediatr Crit Care Med 7: $340-344$

20. Meyer R, Harrison S, Sargent S, Ramnarayan P, Habibi P, et al. (2009) The impact of enteral feeding protocols on nutritional support in critically ill children. J Hum Nutr Diet 22: 428-436.

21. Hamilton S, McAleer DM, Ariagno K, Barrett M, Stenquist N, et al. (2014) A stepwise enteral nutrition algorithm for critically ill children helps achieve nutrient delivery goals. Pediatr Crit Care Med 15: 583-589.

22. Di Girolamo FG, Situlin R, Fiotti N, Biolo G (2017) Intermittent vs. continuous enteral feeding to prevent catabolism in acutely ill adult and pediatric patients. Curr Opin Clin Nutr Metab Care 20: 390-395.

23. Abdelsalam $Y(2012)$ Continuous versus bolus infusion of enteral nutrition in intensive care. AAMJ.

24. Skillman HE, Wischmeyer PE (2008) Nutrition therapy in critically ill infants and children. JPEN J Parenter Enteral Nutr 32: 520-534.

25. Kudsk KA (2007) Beneficial Effect of Enteral Feeding. Gastrointest Endosc Clin N Am 17: 647-662. 
Citation: Alyousef S, Aljassim N, Bashir MS, Yousif M, Mushtaq N, et al. (2017) The Effect of Different Enteral Feeding Protocols on Feeding Tolerance in Critically Ill Children. Int J Pediatr Neonat Care 2: 134. doi: https://doi.org/10.15344/2455-2364/2017/134

Page 9 of 9

26. Ikeda S, Kudsk KA, Fukatsu K, Johnson CD, Le T, et al. (2003) Enteral feeding preserves mucosal immunity despite in vivo MAdCAM-1 blockade of lymphocyte homing. Ann Surg 237: 677-685.

27. M Uozumi, M Sanui, T Komuro, Y lizuka, T Kamio, et al. (2017) Interruption of enteral nutrition in the intensive care unit: a single-center survey. J Intensive Care 5: 52.

28. Kozeniecki M, McAndrew N, Patel J (2016) ICU and process related barriers to optimizing enteral nutrition in a tertiary medical intensive care unit. Nutr Clin Pract 31: 80-85.

29. Kim H, Stotts NA, Froelicher ES, Engler MM, Porter C et al. (2012) Why patients in critical care do not receive adequate enteral nutrition? A review of the literature. J Crit Care 27: 702-713.

30. Mancl EE, Muzevich KM (2013) Tolerability and safety of enteral nutrition in critically ill patients receiving intravenous vasopressor therapy. PEN J Parenter Enteral Nutr 37: 641-651.

31. Chang HR, Bistrian B (1998) The role of cytokines in the catabolic consequences of infection and injury. JPEN 22: 156

32. De Groof F, Joosten KF, Janssen JA, de Kleijn ED, Hazelzet JA, et al. (2002) Acute stress response in children with meningococcal sepsis: importan differences in the growth hormone/insulin-like growth factor I axis between nonsurvivors and survivors. J Clin Endocrinol Metab 87: 3118-3124

33. Btaiche IF, Chan LN, Pleva M, Kraft MD (2010) Critical illness, gastrointestinal complications, and medication therapy during enteral feeding in critically ill adult patients. Nutr Clin Pract 25: 32-49.

34. Premji SS, Chessell L (2011) Continuous nasogastric milk feeding versus intermittent bolus milk feeding for premature infants less than 1500 grams. Cochrane Database Syst Rev 9: CD001819 